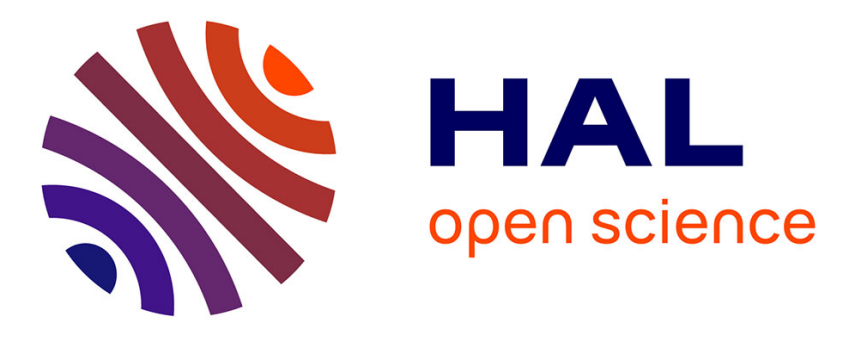

\title{
Radiometric compensation for a low-cost immersive projection system
}

Julien Dehos, Eric Zéghers, Christophe Renaud, François Rousselle, Laurent Sarry

\section{- To cite this version:}

Julien Dehos, Eric Zéghers, Christophe Renaud, François Rousselle, Laurent Sarry. Radiometric compensation for a low-cost immersive projection system. 15th ACM Symposium on Virtual Reality Software and Technology, Oct 2008, Bordeaux, France. pp.130-133, 10.1145/1450579.1450607 . hal00598693

\section{HAL Id: hal-00598693 \\ https://hal.science/hal-00598693}

Submitted on 7 Jun 2011

HAL is a multi-disciplinary open access archive for the deposit and dissemination of scientific research documents, whether they are published or not. The documents may come from teaching and research institutions in France or abroad, or from public or private research centers.
L'archive ouverte pluridisciplinaire HAL, est destinée au dépôt et à la diffusion de documents scientifiques de niveau recherche, publiés ou non, émanant des établissements d'enseignement et de recherche français ou étrangers, des laboratoires publics ou privés. 


\title{
Radiometric compensation for a low-cost immersive projection system
}

\author{
Julien DEHOS* \\ Eric ZEGHERS ${ }^{\dagger}$ \\ LIL, Univ. du Littoral Côte d'Opale \\ LAIC, Univ. d'Auvergne \\ François ROUSSELLE ${ }^{\S}$ \\ Laurent SARRY ${ }^{\top}$ \\ LIL, Univ. du Littoral Côte d'Opale \\ ERIM, Univ. d'Auvergne
}

Christophe RENAUD $\ddagger$

LIL, Univ. du Littoral Côte d'Opale

\begin{abstract}
Catopsys is a low-cost projection system aiming at making mixed reality (virtual, augmented or diminished reality) affordable. It combines a videoprojector, a camera and a convex mirror and works in a non-specific room. This system displays an immersive environment by projecting an image onto the different parts of the room. However, the presence of an uncalibrated projector, heterogeneous materials and light inter-reflections influence the colors of the environment displayed in the room. Radiometric compensation of the projection process enables the system to reduce this problem.

In this paper, we present our low-cost immersive projection system and propose a radiometric model and a compensation method which handle the projector response, surface materials and interreflections between surfaces. Our method works in two stages. First, the radiometric response of the projection process is evaluated. Then, this radiometric response is used to compensate the projection process in the desired environments.
\end{abstract}

CR Categories: I.3.7 [Computer Graphics]: Three-Dimensional Graphics and Realism-virtual reality; I.4.0 [Image Processing and Computer Vision]: General-Image displays; I.4.1 [Image Processing and Computer Vision]: Digitization and Image CaptureRadiometry; I.4.8 [Image Processing and Computer Vision]: Scene Analysis-Photometry;

Keywords: radiometric compensation, mixed reality, virtual reality, immersive projection

\section{Introduction}

In virtual reality frameworks, an immersive environment is a partially or totally artificial environment created around the user. Immersive environments have many applications such as training simulations (flight and driving simulators), architectural or engineering prototyping, virtual offices, entertainment environments, games, art installations... Immersive environments can be displayed by two kinds of systems: wearable devices such as head mounted display devices [Sutherland 1998] and large screen systems such as CAVEs [Cruz-Neira et al. 1993].

Everyday technology makes it possible to develop low-cost mixed reality systems. Catopsys (for catadioptric projection system) is a research project which aims at bringing mixed reality to

*e-mail: julien.dehos@gmail.com

†e-mail: zeghers@iut.u-clermont1.fr

$\ddagger$ e-mail: renaud@lil.univ-littoral.fr

$\S$ e-mail: roussell@lil.univ-littoral.fr

『e-mail: laurent.sarry@u-clermont1.fr

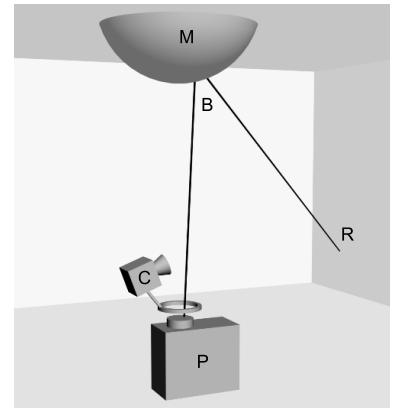

Figure 1: The Catopsys projection system. A projector $(\mathrm{P})$ aims at a convex mirror (M). A beam of light (B) coming from $\mathrm{P}$ is reflected by $\mathrm{M}$ toward the room (R) (wall, floor, ceiling, furniture...). The system can thus display immersive environments. For calibration purposes, a camera (C) can see the whole screen by rotating around the optical axis of $\mathrm{P}$.

the home by developing a low-cost immersive projection system. This system is composed of a projector, a convex mirror and a rotating camera (see Fig. 1). Once in a room, the system calibrates automatically the geometry of its components and of the room [Astre et al. 2008]. Immersive projection can then be done. In [Yuen and Thibault 2008], a system using the same devices is presented. However, it uses a specific screen and, consequently, has different applications.

Our projection system requires radiometric compensation (i.e. color correction). Indeed, since the projection is over non-specific surfaces, images must be modified to really appear as desired, after projection. The projection is influenced by the projector, surface materials and inter-reflections between surfaces. We may notice that inter-reflections are very important for our application because images are projected onto the whole room. To perform radimetric compensation, the system provides a panoramic viewpoint (camera) and a dynamic omnidirectional light source (projector + mirror).

In this paper, we present a radiometric compensation technique adapted to a low-cost immersive projection display. Our method accounts for projector non-linearity, color mixing and interreflections. It has been implemented in a self-calibrating system.

\section{Related work}

Recently, a lot of work has been done to project images faithfully on everyday surfaces [Bimber et al. 2007]. Here, we only consider a few two-stage compensation techniques using a camera-projector system. Such techniques first evaluate the radiometric response of the projection process off-line and then, inverte this response to compute compensation images on-line.

[Nayar et al. 2003] proposes a model of projection on nonspecific screens. The authors also give a calibration/compensation method based on this model. This method separates the projection process into linear color mixing and a per-channel non-linear 
response. However, the screen is supposed to be flat.

[Bimber et al. 2006] proposes a method to compensate projection when inter-reflections occur. The authors present a mathematical and algorithmic formulation of a reverse radiosity approach. However, they do not explain how to get the geometry and the reflectances of the scene automatically. Moreover, they assume the projector response is linear.

Finally, an exhaustive method is proposed in [Wetzstein and Bimber 2007]. This method consists in measuring the light transport matrix i.e. the influence of each projector pixel on each camera pixel. Compensated images can be computed using the inverted matrix. However, the non-linearity of the projector response is not taken into account. Furthermore, the matrix measurement requires a huge number of camera captures.

\section{Catopsys projection system}

The Catopsys projection system aims at democratizing mixed reality. To reach this goal, the system has to be low-cost and achieve immersive projection and self-calibration. Our solution is a catadioptric projector-camera system (see Fig. 1). A convex mirror (M) is fixed to the ceiling of the room. A videoprojector $(\mathrm{P})$ is placed under the mirror and points toward it. Thus, a beam of light (B) emitted by $\mathrm{P}$ is reflected by $\mathrm{M}$ into the room (R). Finally, a wide-angle camera $(\mathrm{C})$ is attached above the projector and oriented toward the walls of the room. The camera can rotate around the optical axis of the projector thanks to a stepper motor. The projector, the camera and the stepper motor are controlled by a computer.

This system fulfills the desired features. First, it is composed of a few low-cost devices. Indeed, over the past few years, projectors have become less and less expensive and can reasonably be bought for home applications. In the same way, affordable models of computer-controlled camera exist. The remaining components of the system are inexpensive. In our implementation, we use a surveillance mirror, a home-made stand for the projector-camera system and a cheap stepper motor which we control through an IEEE 1284 card. In a final product, these elements would be manufactured, which would reduce costs even more.

The system also provides the second desired feature i.e. immersive projection. Indeed, according to the settings of the projector, the position of the mirror and the configuration of the room, the projection can be done onto the whole room ( $4 \pi$ steradians).

Finally, the system is able to automatically calibrate itself. Thanks to the projector-camera combination, the system can project an image and capture the result. More precisely, the system has to be calibrated geometrically (relative positions and orientations of devices, geometry of the room), optically (distortions of optics) and radiometrically (light scattering, spectral responses of devices). In this paper, we deal with radiometric compensation and consider that geometrical and optical calibrations have been done [Astre et al. 2008]. Thus, we know onto which triangle of the room geometry, each pixel is projected. We also have an atomic function which gives, for each pixel of the projected image, the color seen by the camera (see section 6 for examples).

\section{Radiometric model}

\subsection{Projection process}

Consider an image projected by our system (see Fig. 2). On the computer, this image is coded in a color basis. The projector converts it according to its internal color channels and emits the corresponding light. Then, this light strikes screen surfaces (walls, ceiling or floor of the room) and bounces many times between surfaces (inter-reflections). Finally, light is captured by the camera sensors to produce another image, on the computer.

The process involves intra-channel non-linearity (projector and camera responses), linear inter-channel mixing (because projector

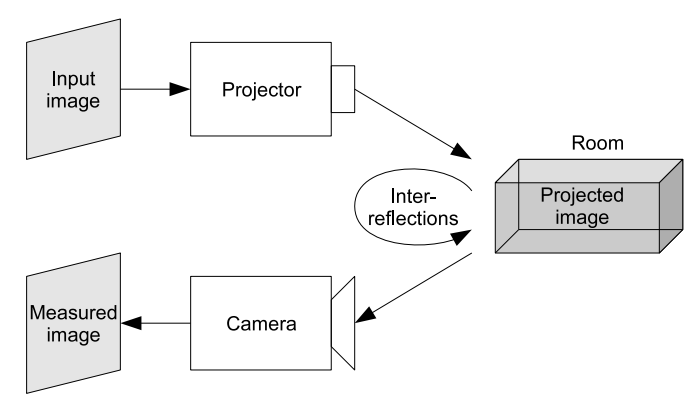

Figure 2: Camera-projector radiometric process.

and camera can have different internal color basis), linear intrachannel scaling (material responses where incident and excitent directions are constant) and linear inter-pixel dependencies (interreflections between surfaces).

\subsection{Radiometric model}

Let us consider a pixel. We call $x$ its color in the linearized color basis of the projector and $y$ its color in the linearized color basis of the camera. Let $t$ be the transition matrix from projector basis to camera basis, then $y=t x$. We define $u$ and $v$ as

$$
u_{i j}=\left\{\begin{array}{ll}
t_{i j}-1 & \text { if } i=j \\
0 & \text { otherwise }
\end{array} \quad, v_{i j}= \begin{cases}1 & \text { if } i=j \\
t_{i j} & \text { otherwise }\end{cases}\right.
$$

Therefore $t=u+v$. We can see $u$ as the intra-channel projectorcamera basis transition. Hence the following radiometric model, partially based on the one given in [Nayar et al. 2003]. Let $a$ be the color of the pixel in the input image (sent to the projector). In the following, we call $x_{\lambda}$ the value of the channel $\lambda$ of the color $x$. The per-channel per-pixel non-linear function $w_{\lambda}$ models the non-linear projector response, the linear material response and $u_{i i}$ (where $i$ is the index corresponding to the channel $\lambda$ in $\mathrm{u}$ ). Let $b$ be the light emitted by the projector and reflected a single time by surfaces of the room, in a scaled linearized projector color basis.

$$
b_{\lambda}=w_{\lambda}\left(a_{\lambda}\right)
$$

Let $c$ be the light after the first reflection, in the linearized camera color basis.

$$
c=v b
$$

We model inter-reflections by the following formula, in linearized camera color basis.

$$
D_{\lambda}=C_{\lambda}+H_{\lambda} C_{\lambda}
$$

$C_{\lambda}$ is the vector containing the $c_{\lambda}$ value of the pixels (or group of pixels) seen by the camera.. It represents the light emitted by surfaces of the room i.e. the light coming from the projector and reflected a single time. $H_{\lambda}$ is a surface-to-surface light exchange matrix, accounting for both geometry and radiometry: $H_{\lambda i j}$ is the part of light emitted from surface $i$, scattered in the room by interreflection and then reflected by surface $j$ toward the camera. Therefore, $C_{\lambda}$ is the light going directly to the camera and $H_{\lambda} C_{\lambda}$ is the light going to the camera after inter-reflection. Finally, $D_{\lambda}$ contains the full light seen by the camera (direct light and interreflections). Consequently, if surface materials are diffuse, light is reflected equally in all directions and equation 3 is valid for any viewpoint. However, if the room contains specular materials, this equation is valid only if the viewpoint is close to the camera.

Finally, the per-channel per-pixel non-linear function $g$ models the camera response. Thus, given a projector pixel, if we call $d$ 
the corresponding light coming from the room (after projection and reflections) toward the camera and $e$ the corresponding color that the camera has captured, we have, for the channel $\lambda$,

$$
e_{\lambda}=g_{\lambda}\left(d_{\lambda}\right)
$$

\section{Compensation algorithm}

In the following, we assume that the camera response is known and corrected. Our radiometric compensation method is composed of two steps. In the first step, the model is evaluated to characterize a specific projection system i.e. to find the $v, w_{\lambda}$ and $H_{\lambda}$ which model the system. In the second step, the evaluated model is inverted to compute compensated images. Once projected, such images are supposed to appear like the desired images.

\subsection{Calibration}

We use the method described in [Nayar et al. 2003] to determine $v$ and $w_{\lambda}$. In the following, we assume that the devices have three channels. Let us consider a pixel. Using the notations of the previous section, we have

$$
\begin{gathered}
a=\left[\begin{array}{l}
a_{r} \\
a_{g} \\
a_{b}
\end{array}\right], b=\left[\begin{array}{l}
b_{r} \\
b_{g} \\
b_{b}
\end{array}\right]=\left[\begin{array}{l}
w_{r}\left(a_{r}\right) \\
w_{g}\left(a_{g}\right) \\
w_{b}\left(a_{b}\right)
\end{array}\right] \\
v=\left[\begin{array}{ccc}
1 & v_{r g} & v_{r b} \\
v_{g r} & 1 & v_{g b} \\
v_{b r} & v_{b g} & 1
\end{array}\right], c=\left[\begin{array}{l}
c_{r} \\
c_{g} \\
c_{b}
\end{array}\right]=v b
\end{gathered}
$$

To determine the elements of $v$, for example $v_{g r}$ and $v_{b r}$, we project two images which only differ in one channel. For example, if we change the red channel of the projected color, we have

$$
a^{(1)}=\left[\begin{array}{c}
a_{r}^{(1)} \\
a_{g}^{(1)} \\
a_{b}^{(1)}
\end{array}\right], a^{(2)}=\left[\begin{array}{c}
a_{r}^{(2)} \\
a_{g}^{(1)} \\
a_{b}^{(1)}
\end{array}\right]
$$

The corresponding captured color $c$ verifies

$$
\left[\begin{array}{c}
c_{r}^{(1)} \\
c_{g}^{(1)} \\
c_{b}^{(1)}
\end{array}\right]=v\left[\begin{array}{c}
b_{r}^{(1)} \\
b_{g}^{(1)} \\
b_{b}^{(1)}
\end{array}\right],\left[\begin{array}{c}
c_{r}^{(2)} \\
c_{g}^{(2)} \\
c_{b}^{(2)}
\end{array}\right]=v\left[\begin{array}{c}
b_{r}^{(2)} \\
b_{g}^{(1)} \\
b_{b}^{(1)}
\end{array}\right]
$$

Thus,

$$
\begin{aligned}
& \Delta c_{r}=\Delta b_{r} \\
& \Delta c_{g}=v_{g r} \Delta b_{r} \quad, v_{g r}=\frac{\Delta c_{g}}{\Delta c_{r}}, v_{b r}=\frac{\Delta c_{b}}{\Delta c_{r}} \\
& \Delta c_{b}=v_{b r} \Delta b_{r}
\end{aligned}
$$

The other unknown elements of $v$ can be computed, in the same way, by changing the green channel and then the blue channel. Since all pixels can be processed in parallel, all $v$ matrices can be computed by projecting only four uniform images.

The function $w_{\lambda}$ can be estimated by projecting every value of $a_{\lambda}$. Indeed, if we project $a_{\lambda}$, we can capture $c_{\lambda}$ and compute $b=v^{-1} c$. Since $b_{\lambda}=w_{\lambda}\left(a_{\lambda}\right)$, this gives $w_{\lambda}$ for $a_{\lambda}$. By projecting gray images we can evaluate $w_{r}, w_{g}$ and $w_{b}$ using the same images. To reduce the number of acquisitions, we carry out a few projection-captures only. The remaining values can then be estimated by interpolation.

This method makes it possible to compensate projection when no inter-reflection occurs. To adapt it to our system, we have to cancel inter-reflections in captured images. To achieve that, we capture direct illumination only, by using the technique proposed in [Nayar et al. 2006]. This technique enables the system to separate direct and indirect illumination of a scene uniformly lit by a projector. Basically, it consists in projecting a checkerboard. Thus a point of the room corresponding to a pixel in a black square of the checkerboard is not directly lit. Therefore, the corresponding light captured by the camera is due to indirect lighting only. By translating the checkerboard, we can get the indirect light for every pixel. Finally, we can get the direct light for a pixel by considering a colored square. Here, the captured light is the sum of direct light and indirect light. Since we have characterized indirect light, we can get direct light by subtraction.

The last calibration step aims at determining $H_{\lambda}$ where $H_{\lambda i j}$ is the part of light (of the channel $\lambda$ ) leaving $i$ which is scattered in the room and then reflected by $j$ toward the camera. [Sen et al. 2005] proposes a hierarchical method to determine such a matrix. Basically, this method works by considering pixel pairs i.e. it determines the contribution of each projector pixel onto each camera pixel. In our implementation, we simply group pixels which are projected onto the same triangles of the room geometry.

Thus, to determine $H_{\lambda i j}$, we project an image where all pixels matching the triangle $i$ are white (other pixels are black). Knowing the projected image and a part of the projection response ( $v$ and $w_{\lambda}$ ), we compute the light which is effectively emitted by the triangle $i$ in the room. This enables the system to normalize the captured image. By summing the value of all normalized captured pixels matching the triangle $j$, we obtain $H_{\lambda i j}$.

\subsection{Compensation}

First, we have to compensate for inter-reflections. Equation 3 is equivalent to

$$
C_{\lambda}=F_{\lambda} D_{\lambda}
$$

where

$$
F_{\lambda}=\left(I+H_{\lambda}\right)^{-1}=I-H_{\lambda}+H_{\lambda}^{2}-\ldots
$$

Equation 10 gives the light each triangle should emit to compensate for inter-reflections. Since this equation considers triangles, we have to set a relation between pixels and triangles. We obtain the value of a triangle in $D_{\lambda}$ by summing the value of the corresponding pixels. Then, we compute equation 10. Finally, we can compensate for inter-reflections in the image by weighting each pixel with the value of the corresponding triangle in $C_{\lambda}$. However, this will show discontinuities between triangles. Therefore we use the classic Gouraud method [Gouraud 1998] to take back the values from triangles to pixels. This gives an image that compensates for inter-reflections.

Finally, we have to inverte equation 2 and equation 1 with

$$
b=v^{-1} c, \quad a_{\lambda}=w_{\lambda}^{-1}(b)
$$

Projecting pixel colors $a$ should make the camera see the desired colors $d$. This radiometrically compensates the projection system.

\subsection{Implementation}

Unlike calibration, compensation has to be computed for every image to project. Therefore, it must be done as quickly as possible. By precomputing some steps $\left(v^{-1}\right.$ and $\left.F\right)$ during calibration, we can reduce compensation computations. Consider we use RGB color spaces. Let $T$ and $N$ be the number of triangles and the number of pixels, respectively. Since $F$ has been precomputed, the first compensation step requires three products of a $T \times T$ matrix by a $T$ vector and Gouraud smoothing. Similarly, $v^{-1}$ has been precomputed, therefore the second step requires the product of a $3 \times 3$ matrix by a 3 vector, for each pixel (i.e. $N$ products). Finally, for each pixel, compensating for $w_{\lambda}$ resumes to invert a sparsely discretized function i.e. find the neighboring samples and interpolate inverse values. 


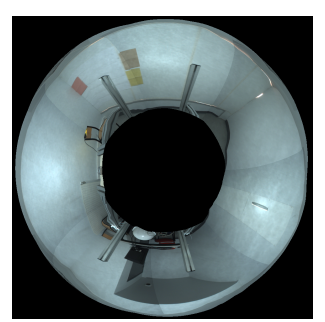

(a)

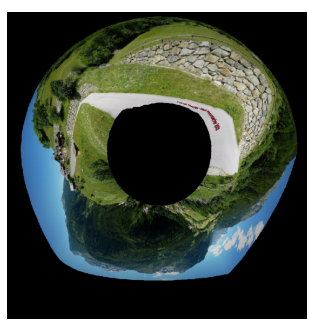

(b)

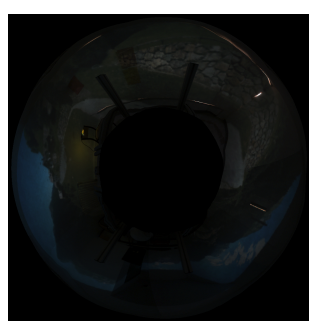

(c)

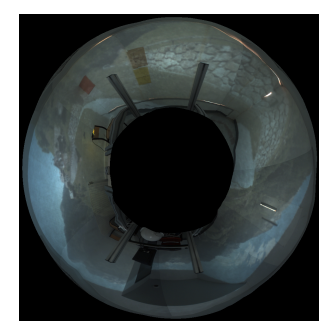

(d)

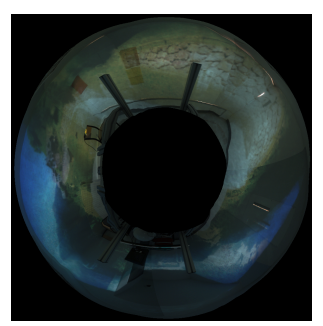

(e)

Figure 3: Compensation results for a virtual reality application. (a) room. (b) desired image. (c) uncompensated projection (error $=0.064$ ). (d) uniformly compensated projection (error $=0.054)$. (e) compensated projection (error $=0.049$ ).

\section{Results}

We have tested these algorithms with our system (SXGA DLP projector, XGA 3-CCD camera). Our test room is composed of specular white-painted walls onto which are hung sheets of colored paper. The calibration step requires about ten hours. This time is almost exclusively devoted to image acquisition, since the number of required captures is very significant. Compensation can be done quickly (about 5 images per second), using the described precomputations. We call error of a captured image, the evaluation of $\frac{1}{N} \sum_{p}\left\|c_{p}-\hat{c}_{p}\right\|_{2}^{2}$ where $N$ is the number of pixels and $c_{p}$ (resp. $\hat{c}_{p}$ ) the Luv color of the pixel $p$ in the desired image (resp. in the captured image).

Fig. 3 presents some of our results. These images give for each pixel of the projector, the color seen by the camera. Our test room (a) has specular material and colored area. The desired image (b) is a panoramic landscape. Without compensation (c), the projected environment is unacceptably too dark. A basic uniform compensation (d) makes the environment become more visible. However, we can still notice the materials of the room (colored area, specular highlights) and the projector response (color shifts, contrast attenuation). Our method (e) compensates these phenomena and manages to display an environment which is close to the desired one.

\section{Conclusion and future works}

In this paper, we have presented the Catopsys projection system. This system combines a videoprojector, a mirror and a rotating camera. It is low-cost, self-calibrating and performs immersive projection. Then, we have given a radiometric model of the projection process. This model accounts for non-linearity, color mixing and inter-reflections. Finally, we have given a calibration algorithm and a compensation algorithm, based on this model, which make it possible to compensate the projection process.

In future works, we will try to improve the accuracy of the method. Using high dynamic range images should avoid camerasensor saturation and thus extends the range of colors which can be compensated for. Using a perceptually-uniform color space, such as Luv, may also give more faithful results. Finally, we will try to explicitly characterize the materials of the scene to account for specular components.

\section{Acknowledgements}

This work is founded by the Audiovisual and Multimedia Program of the French Agency for Research (Catopsys project). The authors are grateful to Christophe LOHOU and Susan ARBON for help in preparing this paper.

\section{References}

Astre, B., SArry, L., Lohou, C., And Zeghers, E. 2008. Automatic calibration of a single-projector catadioptric display system. In IEEE Conference on Computer Vision and Pattern Recognition (CVPR).

Bimber, O., Grundhofer, A., Zeidler, T., DANCh, D., AND KAPAKOS, P. 2006. Compensating indirect scattering for immersive and semi-immersive projection displays. In $V R$ '06: Proceedings of the IEEE conference on Virtual Reality, IEEE Computer Society, Washington, DC, USA, 151-158.

Bimber, O., Iwai, D., Wetzstein, G., And GrundhöFer, A. 2007. The visual computing of projector-camera systems. In EuroGraphics (STAR).

Cruz-Neira, C., Sandin, D. J., And DeFanti, T. A. 1993. Surround-screen projection-based virtual reality: the design and implementation of the cave. In SIGGRAPH '93: Proceedings of the 20th annual conference on Computer graphics and interactive techniques, ACM, New York, NY, USA, 135-142.

Gouraud, H. 1998. Seminal graphics: poineering efforts that shaped the field. ACM, New York, NY, USA, ch. Continuous shading of curved surfaces, 87-93.

Nayar, S., Peri, H., Grossberg, M., and Belhumeur, P. 2003. A Projection System with Radiometric Compensation for Screen Imperfections. In ICCV Workshop on Projector-Camera Systems (PROCAMS).

Nayar, S. K., Krishnan, G., Grossberg, M. D., And RASKAR, R. 2006. Fast separation of direct and global components of a scene using high frequency illumination. ACM Trans. Graph. 25, 3, 935-944.

Sen, P., Chen, B., Garg, G., Marschner, S. R., Horowitz, M., Levoy, M., AND LensCH, H. P. A. 2005. Dual photography. ACM Trans. Graph. 24, 3, 745-755.

SUTHERLAND, I. E. 1998. Seminal graphics: poineering efforts that shaped the field. ACM, New York, NY, USA, ch. A headmounted three dimensional display, 295-302.

Wetzstein, G., AND Bimber, O. 2007. Radiometric compensation through inverse light transport. In $P G$ '07: Proceedings of the 15th Pacific Conference on Computer Graphics and Applications, IEEE Computer Society, Washington, DC, USA, 391-399.

Yuen, N. P. Y., And Thibault, W. C. 2008. Inexpensive immersive projection. In $V R, 237-240$. 